INVESTIGACIONES

\title{
El profesor y su identidad profesional ¿facilitadores u obstáculos del cambio educativo?
}

\author{
The teacher and his professional identity. Facilitators or \\ obstacles to educational change? \\ O professor e sua identidade profissional: facilitadores \\ ou obstáculos para a transformação educacional?
}

\author{
Alberto Galaz. \\ Facultad de Filosofía y Humanidades, Universidad Austral de Chile. \\ (56-63) 221262, alberto.galaz@uach.cl
}

\begin{abstract}
RESUMEN
La adopción de enfoques por competencias, la formulación de estándares de desempeño y la implementación de sofisticados sistemas de evaluación expresan la preocupación de las autoridades por comprometer a los profesores en el mejoramiento de la calidad de la educación. Sin embargo, el perfil del profesor subyacente a los cambios puede no necesariamente ser coincidente con la definición que los profesores construyen de si, lo que puede contraer consecuencias a nivel de su reconocimiento profesional y constituirse por consiguiente en un obstáculo para el mejoramiento deseado. A través de una aproximación metodológica que combina la aplicación de un cuestionario a profesores de secundaria y el análisis documental del discurso oficial, este artículo identifica los encuentros y desencuentros que se producen entre la identidad promovida y la identidad construida. Se discuten además las condiciones que favorecerían la efectividad de las innovaciones.
\end{abstract}

Palabras clave: competencias, identidad, modelos identitarios, profesores y políticas.

\begin{abstract}
The trend towards focusing on educative competences, the creation of performance standards and the implementation of sophisticated systems of evaluation, has raised concern amongst authorities to commit the professors towards improvement of the quality of education. However, the profile of the underlying professor towards the previously mentioned changes does not necessarily coincide with the definition that professors have already constructed. This may produce consequences concerning ones' professional recognition and thus constitute an obstacle towards ones' desired improvement as a professor. Through an approximate methodological approach that combines the application of a questionnaire to high school professors and the documentary analysis of the official discourse from these professors, this article identifies the understandings and misunderstandings that take place between the developing identity and the constructed identity. In addition, the conditions that would favor the effectiveness of such innovations are discussed.
\end{abstract}

Key words: competences, identity, identity models, professors and policies.

\section{RESUMO}

A adoção de enfoques de concorrências, a construção de padrões de desempenho e a implementação de sofisticados sistemas para avaliar expressam a preocupação das autoridades por comprometer aos professores no melhoramento da qualidade da educação. No entanto, a imagem promovida do professor pode não coincidir com a imagem que os professores constroem de si mesmos, o que pode se constituir em problemas no reconhecimento profissional e, consequentemente, em obstáculo para o melhoramento desejado. Por meio de uma aproximação metodológica que combina um questionário respondido por professores do Ensino Médio e a análise documental, este artigo identifica diferenças e semelhanças entre a identidade promovida e a identidade construída. Discutemse também as condições que favoreceriam a efetividade das inovações.

Palavras-chave: competências, identidade, modelos identitários, professores e políticas. 


\section{INTRODUCCIÓN}

La insatisfacción existente con los resultados obtenidos en las recientes pruebas de medición de la calidad de los aprendizajes de los estudiantes, ha llevado al Ministerio de Educación de Chile (Mineduc) a identificar la formación y el desempeño de los profesores como áreas prioritarias para la promoción y evaluación de competencias, en el convencimiento de que esta iniciativa podrá incidir efectivamente en el mejoramiento. Si bien la noción de competencia ha sido empleada en el discurso oficial desde fines de la década de los 90', ha sido sin embargo desde principios del año 2000 que se ha instalado como un enfoque orientador de las normativas educativas, por ejemplo a nivel de la definición de los ámbitos de desempeño presentes en el Marco para la Buena Enseñanza (Mineduc, 2003), en la aprobación de reglamentos para la Evaluación del Desempeño de los profesores en el 2004 y, más recientemente, a través de un Programa de Mejoramiento de la Calidad de la Educación Superior (MECESUP) y en la evaluación y definición de estándares específicos para la formación inicial de profesores, esta última bajo un programa denominado "Inicia".

Con estas medidas las autoridades educativas esperan formar profesionales, colaboradores, autónomos y constructores de conocimiento. Aunque un análisis más exhaustivo del discurso permite revelar una imagen más acotada, la de un profesor que, consciente de la incidencia de su práctica en los resultados de sus alumnos, activa sus competencias para dar cuenta de las diferencias entre lo planificado y lo efectivamente logrado. Es decir, profesores que cuentan con las competencias que se correlacionan significativamente con el logro efectivo de los aprendizajes. Al respecto, Fullan (2002) y Tardif (2004) han constatado en sus análisis de las reformas educativas en Estados Unidos y Europa la existencia de similares referencias y han coincidido que, en un terreno más concreto, el objetivo central que se plantean los gobiernos es formar profesores que realizan un permanente proceso de mejora de sus competencias.

De esta forma, las políticas diseñadas para promover, implementar y evaluar competencias pueden ser concebidas como apuestas educativas y pedagógicas que buscan promover nuevas formas de ser y hacer (Day et al. 2005; Maroy, 2001). Al respecto, Díaz (2001) señala que estas apuestas ocurrirían en circunstancias en que hay posibilidades para la "desarticulación de una identidad" y supone la proposición de "una identidad o de un modelo de identificación distinto" que busca impregnar de nuevos significados y dinámicas el desempeño. Sin embargo, otros estudios han planteado que una identidad profesional no se asume automáticamente. Las evidencias se dirigen a demostrar la existencia de estrategias personales tendientes a rechazar, cambiar o simular las identidades promovidas en virtud de las propias historias y concepciones (Cattonar, 2001; Maroy, 2001; Woods, 2002; Czop, 2008; Hong, 2010; entre otros). Un aspecto interesante de estas referencias es que permiten apreciar que la identidad profesional se constituye en un factor importante para la comprensión de la vida profesional del profesor (Beauchamp, 2009), porque el concepto que él construye de sí y de su profesión es una base sobre la cual proyecta su desarrollo y su compromiso con el cambio o mejoramiento educativo (Day, 2010; Lasky, 2005 y Astolfi, 2003).

Sin embargo, el alto nivel de aceptación que ha tenido el enfoque por competencias por parte de las autoridades educativas y las instituciones formadoras de profesores se opone a la escasa investigación existente sobre la efectividad de las estrategias empleadas para 
desarrollarlas y sus impactos sobre la identidad de los profesores. Entonces, es posible que las políticas educativas en general y aquellas de formación de profesores en particular pudieran estar promoviendo competencias que no necesariamente son coincidentes con la identidad que construyen los profesores. La incertidumbre generada conlleva riesgos asociados a la eficiencia y efectividad de las propuestas de cambio, pero así también, a nivel del reconocimiento de la persona del profesor, su identidad y su labor. Por tales antecedentes cabe preguntarse: ¿qué identidad profesional del profesor se promueve a nivel oficial? y ¿qué competencias promueven?, ¿cuál es la identidad que construyen los profesores? y ¿qué competencias son las que emplean efectivamente en su trabajo?, ¿qué proyecciones pueden desprenderse de las diferencias y semejanzas identitarias a fin de promover un efectivo mejoramiento educativo?

Los estudios referidos a la identidad profesional de los profesores chilenos han estado principalmente referidos a los conceptos o imágenes que desarrollan sobre sí mismos, de esta forma, poseen la limitación de abordar primordialmente la identidad desde una dimensión subjetiva y, en menor medida, desde los complejos procesos de interacción y de identificación. Por último, cabe señalar que, a diferencia de los profesores en formación y aquellos de enseñanza primaria, las particularidades de las construcciones identitarias que desarrollan los profesores de secundaria actualmente en servicio no han constituido un foco de mayor interés para los investigadores. Por tales motivos, este estudio pretende identificar comparativamente la identidad construida por los profesores de secundaria pertenecientes a tres liceos y aquella que subyace a la promoción y evaluación oficial de competencias contenidas en las políticas educativas. Nuestro interés ha sido develar las similitudes o diferencias, encuentros o desencuentros, porque los hallazgos podrían servir como indicadores de compromiso sobre las innovaciones y como una matriz para el análisis y evaluación de la efectividad de las estrategias adoptadas para el mejoramiento de la educación.

\section{LA IDENTIDAD PROFESIONAL: UN PROCESO DE CONSTRUCCIÓN COMPLEJO Y DINÁMICO}

La noción "identidad profesional" alude a una definición en referencia a un campo o área específica de desempeño. Es una definición que según Beijaard (2004), Cattonar (2001), Gohier (2000) y Vonck (1995) entre otros, debe ser concebida aludiendo a un proceso dinámico e interactivo de construcción, en el cual confluyen variables de carácter tanto subjetivo (la historia personal) como social (las referencias y pertenencias colectivas). La identidad profesional puede ser entendida además como la síntesis de un proceso de identificación o construcción de la identidad en virtud de modelos de referencia, y otro de identización o consideración de elementos de diferenciación de naturaleza biográfica o personal. Según Dubar (2000), al ser puestos estos procesos en juego, los profesionales se dotan de un discurso legitimante cuyos contenidos apuntan a evidenciar la presencia de contenidos tales como: competencias, valores, desafíos, etc. que poseen un carácter particular y privativo y que permiten el dominio (percepción de sí) y el reconocimiento (social/oficial).

Planteamos también que un profesor posee tantas identidades o formas de definirse como sean los ámbitos de su socialización (Cattonar, 2001; Maroy, 2001; Czop, 2008 y Hong, 2010). Woods (2002); por ejemplo, alude a la existencia de "identidades sustanciales" 
y "situacionales" estableciendo un marco de diferencias en términos de duración, resistencia y posicionamiento. La primera de ellas alude a los elementos definitorios que permanecen independientes de todo contexto, tiempo e inclusive inmunes a toda asignación, y que por el contrario, en su interacción ellos parecen reafirmarse. La segunda define la instalación de elementos transitorios, que son constantemente modificados en virtud, como hemos señalado, de los contextos de socialización.

Ante esta diversidad cabe preguntarse ¿cómo el profesor puede mantener una mínima continuidad y coherencia profesional? Nuestra proposición es muy cercana a la de Tardif (2004). Constatada la existencia de una identidad profesional plural, como así también la fuerza y significatividad que adquieren algunos elementos o contenidos en su construcción, es posible establecer que ella puede ser mejor concebida como formando un núcleo caracterizado por su fuerte grado de transversalidad y por ser capaz de dotar de significado o sentido a aquellas identidades de carácter periférico.

Según Cattonar (2001), pueden ser dos los conjuntos periféricos asociados al núcleo de la identidad profesional: las identidades profesionales vinculadas a los niveles de enseñanza y las identidades profesionales flexibles, éstas últimas vinculadas a los efectos del contexto de trabajo y las variaciones individuales. Por nuestra parte, si bien compartimos la existencia de un núcleo y de una periferia, estimamos que el contenido hipotético del primero es diversificado y complejo, y que en el caso de los profesores de secundaria no solo está dado por su fuerte referencia a la disciplina enseñada, sino también por aquellas competencias de naturaleza emocional, relacional y comunicacional.

Todo depende del marco desde el cual se realiza el análisis. Por ejemplo, si la evocación de la identidad se realiza desde el profesor, el núcleo de aquella identidad poseerá un contenido de fuerte carácter personal y contextual. Los contenidos de referencia por cierto pueden poseer distintos grados de transversalidad con el colectivo de todos los profesores y los profesores de la misma disciplina, nivel de enseñanza o institución específica, lo cual significa que el núcleo puede variar su eje según el contenido al cual esté asociado.

Pero la centralidad puede ser concebida como el mayor riesgo para la identidad, porque sea producto de atribuciones o demandas de gran persistencia, fuerza e implicantes de cambios, a nivel contextual puede derivar en una crisis. Un núcleo puede ser así transformado, específicamente cuando las nuevas prácticas o las transformaciones del contexto cuestionan su significación central y crean una incoherencia insoportable que no deja más remedio que la transacción.

Finalmente, y en base a los antecedentes analizados, estamos en condiciones de establecer que la identidad profesional de los profesores es una construcción que se realiza a lo largo de toda la vida del profesor, en sucesivas etapas y eventos complejos de clasificar, y que la imagen o concepto que el profesor construye de sí como profesional reúne dimensiones afectivas y cognitivas, personales y sociales en torno a una constante proyección al pasado y al futuro.

\section{COMPETENCIAS PROFESIONALES}

Como resultado de su empleo en diversos campos disciplinares y los diferentes significados que por tal motivo adquiere, la noción de competencia puede ser mejor definida por su carácter polisémico y dinámico. 
Figura 1

Núcleo hipotético de la identidad profesional del profesor

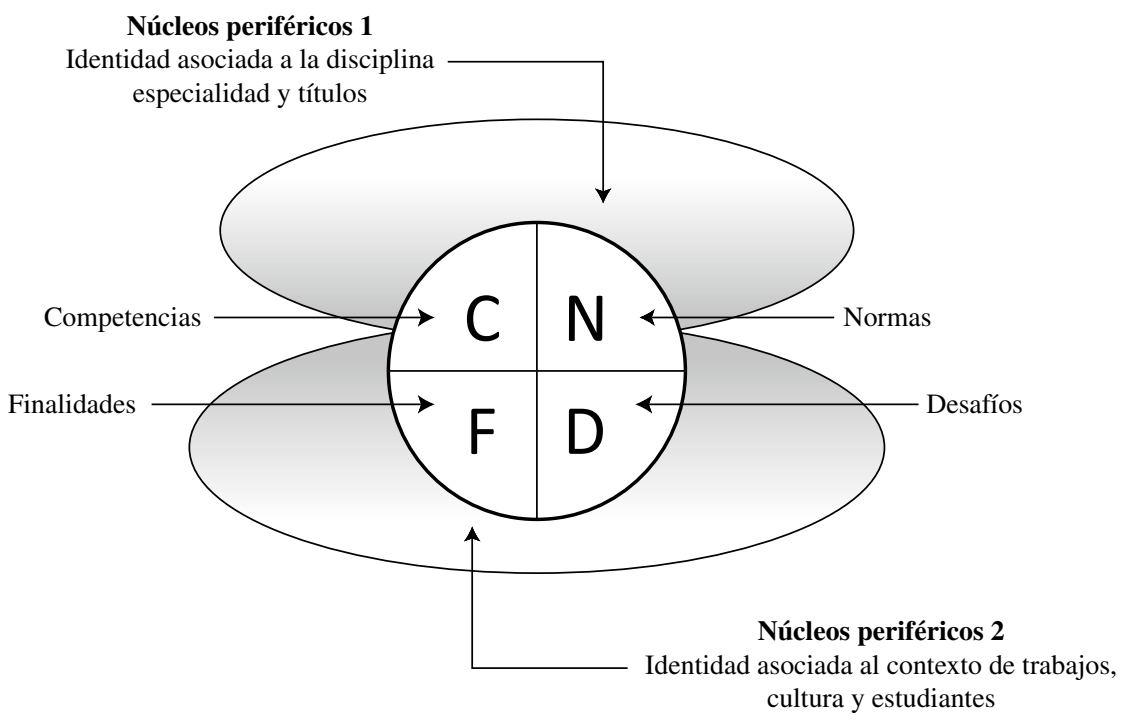

Paquay (2001) por ejemplo, señala que una competencia hace referencia a un conjunto diversificado de saberes cognitivos, afectivos, conativos y prácticos. Si bien concuerdan con esta diversidad, otros autores prefieren aludir a saberes de carácter conceptual, procedimental y actitudinal (Letor, 2003). Para Desjardins (2003) y Tardif (2008), sin embargo no se trata tan solo de contar con saberes sino más bien de que éstos deben ser empleados en la resolución de problemas asociados al ejercicio profesional. A juicio de estos autores, esta dinámica permite posicionar reflexivamente al profesor con el fin de que evalúe y seleccione sus recursos.

Establecidos sus alcances, cabe preguntarse: ¿cuáles son las posibilidades o ventajas de la adopción de un enfoque de competencias en el campo de la educación? y ¿cuáles son sus implicancias identitarias o profesionales?

Respecto a la primera pregunta autores como Astolfi (2003), señalan que ella:

a. Posibilita dar cuerpo a un conjunto de capacidades procedimentales.

b. Se vincula a un contexto determinado y a situaciones concretas de desempeño.

c. Permite integrar diferentes tipos de capacidades que se combinan entre sí para permitir la consecución de los objetivos.

Respecto a sus implicancias identitarias, cabe señalar que en el marco de desarrollo histórico de toda profesión, la formación y el empleo de competencias es un asunto crucial, porque en virtud de su efectiva posesión el profesional se define como categoría social. $\mathrm{Su}$ gravitación en el proceso de construcción identitaria por lo tanto es fundamental por dos razones: en primer lugar, porque forma parte central del núcleo de la identidad del profesional (Tardif y Lessard, 1999), y en segundo lugar, porque como advierte Dubar 
(1991), la posibilidad de disminuir la distancia o diferencia entre la imagen personalmente construida por el profesional y aquella que asignan las autoridades, surge del reconocimiento o no reconocimiento de las competencias que se han promovido y adquirido a lo largo del ciclo de vida o carrera profesional (Bolívar, 1999).

Es importante señalar brevemente que, entre otras clasificaciones, existen tres corrientes de comprensión, construcción y determinación de competencias profesionales. Estas son:

a. La funcionalista. Define las competencias como normas de rendimiento convenidas y desarrolladas por el sector productivo. Su determinación se realiza en base a resultados o rendimientos reales.

b. La conductista. Coloca su acento en una determinación de competencias en base a investigaciones que se realizan sobre "ejecutores expertos".

c. La constructivista. Alude a las competencias como resultantes de procesos de aprendizaje situado y en el cual se incorpora a toda la población.

\section{Figura 2}

Enfoques de competencias

\begin{tabular}{|l|l|l|}
\hline Funcional & Conductista & Constructivista \\
\hline $\begin{array}{l}\text { Normas de rendimiento de- } \\
\text { sarrolladas convenidas por la } \\
\text { industria. }\end{array}$ & $\begin{array}{l}\text { Grupos de competencia desarro- } \\
\text { llados por investigación basada } \\
\text { en excelentes ejecutores. }\end{array}$ & $\begin{array}{l}\text { Competencias desarrolladas por } \\
\text { procesos de aprendizaje ante } \\
\text { disfunciones y que incluye a la } \\
\text { población menos competente. }\end{array}$ \\
\hline $\begin{array}{l}\text { Normas basadas en resultados } \\
\text { (referencia a criterio). }\end{array}$ & $\begin{array}{l}\text { Normas orientadas a resultados } \\
\text { (validadas por criterio). }\end{array}$ & $\begin{array}{l}\text { Normas construidas a partir de } \\
\text { resultados de aprendizaje. }\end{array}$ \\
\hline $\begin{array}{l}\text { Normas de competencia ocu- } \\
\text { pacional (rendimiento real en } \\
\text { el trabajo). }\end{array}$ & $\begin{array}{l}\text { Proceso educacional (desarrollo } \\
\text { de competencia). }\end{array}$ & $\begin{array}{l}\text { Procesos de aprendizaje por } \\
\text { alternancia en planta. }\end{array}$ \\
\hline $\begin{array}{l}\text { Fijación de rendimiento compe- } \\
\text { tente, convenida sectorialmene. }\end{array}$ & $\begin{array}{l}\text { Especificaciones de rendimiento } \\
\text { superior definido por investiga- } \\
\text { ción educativa. }\end{array}$ & $\begin{array}{l}\text { Especificaciones definidas por } \\
\text { los alcaldes logrados en plantas } \\
\text { por los trabajadores. }\end{array}$ \\
\hline Producto: competencias duras. & $\begin{array}{l}\text { Producto: competencias } \\
\text { blandas. }\end{array}$ & $\begin{array}{l}\text { Producto: competencias } \\
\text { contextuales }\end{array}$ \\
\hline
\end{tabular}

Fuente: Cidec, 1999.

Por último, cabe advertir sobre los riesgos de adopción de un enfoque de competencias bajo definiciones únicas y sin prestar atención a las consecuencias identitarias (2010). Ante la multiplicidad de enfoques existentes, las consecuencias de tal decisión pueden ser la exclusión, la incertidumbre y la falta de pertinencia o legitimidad de las propuestas. 


\section{MODELOS IDENTITARIOS}

La definición de modelo recoge los aportes de los autores Berger y Luckmann (1992), quienes señalan que un modelo puede ser entendido como un esquema de referencia o de tipificación preexistente, estable y accesible. Sirven por lo tanto como recursos y estrategias para identificar o identificarse colectivamente, aunque también como referente para revelar los elementos propios de la identidad de los profesionales. En el campo de la educación y formación de profesores, Zeichner (1993 y 1999), Kelchtermans (2001) y Maroy (2001 y 2005) han identificado la presencia de al menos tres modelos profesionales (técnico, práctico reflexivo y crítico-reflexivo) de base para la identidad de los profesores. A continuación se detallan algunas de sus características y competencias asociadas.

\subsection{MODELO TÉCNICO}

Su concepto central es el de "competencia", entendida como aquellas acciones posibles de ser observadas y que resultan ser efectivas al momento de la medición y/o evaluación de los logros. Su presupuesto es que la enseñanza puede ser mejorada utilizando los resultados de la investigación y experimentación educacional En este contexto, el profesor asume un rol pasivo en materia de diseño de programas y estrategias de enseñanza.

\subsection{MODELO PRÁCTICO REFLEXIVO}

Este modelo tiene sus orígenes en los aportes de Schön (1983). Sostiene que los profesores no son sólo 'aplicadores' o 'implementadores' de teorías o soluciones estandarizadas, sino que ellos resignifican, interpretan y construyen su saber profesional. Esta capacidad reflexiva les permite actuar de forma creativa sobre el contexto educativo y asumir una posición protagónica sobre su desarrollo profesional.

\subsection{MODELO CRÍTICO REFLEXIVO}

Según sostienen Zeichner (1993 y 1999) y Kelchtermans (2001), la reflexión es una práctica social emancipadora que implica develar los supuestos ideológicos de los programas y la implementación de estrategias que favorezcan el desarrollo de posiciones crítico-constructivas ante el conocimiento y los condicionamientos sociales.

\section{METODOLOGÍA}

La investigación se orientó fundamentalmente por un diseño cualitativo, pero que en dimensiones específicas empleó análisis descriptivos de naturaleza cuantitativa. De esta forma se busca combinar métodos con el objetivo de que se produzca una convergencia de sus potencialidades según sugieren Hong (2010) y Johnson y Turner (2003).

Para dar cuenta de las interrogantes y objetivos formulados se ha tenido en consideración la necesidad de identificar los contenidos (competencias, normas, desafíos y finalidades) del núcleo de la identidad promovida por las políticas, como así también, aquellos que constituyen la identidad de los profesores de secundaria. Las identidades resultantes son analizadas y contrastadas. Dos fases de investigación fueron planificadas para dicho efecto: 
Las acciones estuvieron dirigidas a describir y analizar las iniciativas políticas educativas que permitían establecer el modelo profesional que sirvió de referencia y los contenidos específicos de la identidad profesional que se demanda a los profesores de secundaria.

\section{Fuentes documentales}

La estrategia desarrollada implicó, en primer lugar, la búsqueda de documentos de carácter oficial (reportes evaluativos o investigativos) y publicaciones que pueden ser consideradas como fuentes, referentes o portavoces oficiales del discurso o propuesta educativa oficial. Sobre la base de una previa selección de 36 documentos, que van desde mediados de los 90' al 2009 se ha realizado, en segundo lugar, un análisis de contenido que ha permitido dar cuenta de las categorías identitarias asociadas. La recolección, clasificación y sistematización del contenido de la documentación se ha hecho en base a una Matriz de Registro.

\section{Estrategia de análisis}

La construcción e interpretación de las categorías subyacentes al discurso educativo oficial se realizó con la técnica cualitativa del análisis de contenido. Tres etapas fueron consideradas en este análisis.

\section{a. Exploración}

Se extrajo bajo principios o ejes generales preestablecidos de búsqueda aquellos significados a partir de los cuales es posible desarrollar categorías inclusivas.

\section{b. Descripción}

Consistió en la realización de examen de todos los significados en cada categoría a fin de establecer patrones en los datos. Para ello fue necesaria la construcción de matrices a partir de las cuales se pudo desprender y clasificar las categorías para su posterior interpretación.

\section{c. Interpretación}

Una vez extraídas y clasificadas las categorías, se procedió a la búsqueda de sus conexiones y sus relaciones con el conjunto de datos (cuando convergían hacia un mismo significado) o, por otra, las oposiciones en sus distintos niveles (fundamentalmente a nivel individual o grupal). Una vez identificadas, clasificadas y analizadas las categorías, se procedió a su interpretación bajo los principios teóricos que sostienen el marco conceptual del estudio.

\section{FASE 2. IDENTIDAD PROFESIONAL CONSTRUIDA POR LOS PROFESORES DE SECUNDARIA}

En esta fase las acciones se dirigieron a obtener información sobre los modelos, contenidos y factores que sirven como referentes de identificación para las identidades 
que construyen los profesores. La estrategia desarrollada se dirigió, en primer lugar, a la búsqueda de los antecedentes profesionales (disciplina enseñada, experiencia, institución de formación, etc.) que permitieran formar un marco de interpretación relativo a sus pertenencias y trayectorias.

En segundo lugar, se buscó establecer la forma en que los profesores sintetizan sus competencias profesionales, y en tercer lugar, se identificaron los eventos y factores que pueden ser considerados como ejes explicativos del desarrollo de las identidades.

La recopilación de información se realizó por medio de un Cuestionario de Identidad Profesional construido a partir de los aportes de la literatura que ha estado referida a la identificación de modelos identitarios, sus dimensiones, contenidos, procesos y fases involucradas. Así también, recoge las experiencias de aplicación de instrumentos similares realizadas por Woods (2002), Beijaard (2002 y 2004), Cattonar (2001) y Maroy (2001), entre otros. Para validar el instrumento se estableció su consistencia interna. Para tal efecto se utilizó como medida el Alfa de Cronbach obteniendo como resultado un coeficiente confiabilidad general de 0,86 y un coeficiente 0,87 por cada ítem.

El cuestionario final consta de las siguientes dimensiones:

Parte I. Antecedentes socioeconómicos, formativos y profesionales del profesor.

Parte II. Referentes o contenidos fundamentales de la identidad profesional.

Parte III. Modelo profesional de referencia.

Parte IV. Eventos y factores significativos en la construcción de su identidad profesional.

\section{Participantes}

Un total de 53 profesoras y 13 profesores $(n=64)$ de secundaria pertenecientes a tres liceos con financiamiento público de la Región Metropolitana de Santiago fueron seleccionados para esta fase de investigación. La alta participación de profesoras en relación a los profesores puede ser concebida como representativa del actual proceso de feminización del cuerpo de profesores en Chile. Por otra parte, y dado que se trata de factores esenciales en el análisis de la construcción de la identidad profesional, los criterios de selección de los profesores obedecieron a dos razones: en primer lugar a la disciplina enseñada (Artes, Ciencias, Lenguaje, Ciencias Sociales, Matemáticas, Tecnología y Educación Física) y, en segundo lugar, a los años de experiencia (Huberman, 1989 y 1990). Al respecto, los participantes contaban en promedio con 15 años, dentro de un rango de 1 y más de 30 años.

\section{Estrategia de análisis}

El análisis de la identidad de los profesores se realiza en consideración del número total de participantes. Las respuestas de los profesores son clasificadas en virtud de su total o nula identificación (Escala Likert de 4 alternativas) con las características profesionales contenidas en cada afirmación. Los análisis estadísticos son de carácter descriptivo. Se ha recurrido, además, a pruebas estadísticas para sostener la validez de los resultados obtenidos. 


\section{RESULTADOS OBTENIDOS}

\subsection{IDENTIDAD PROFESIONAL PROMOVIDA POR LAS ACTUALES POLÍTICAS EDUCATIVAS}

El análisis de la documentación oficial ha permitido identificar tres ejes de categorías en torno a los cuales se articulan los significados de la identidad del profesor que se desea promover a través del discurso educativo oficial. Estos son:

\section{a. Categoría $N^{o}$ 1. Profesionalismo del profesor}

Las políticas educativas dirigidas al profesor se desarrollan sobre tres vertientes. La primera es la de "fortalecimiento de la profesionalidad". Esta vertiente será eje fundamental de la Reforma Educacional del año 1996, bajo la denominación de Desarrollo Profesional Docente (MINEDUC, 1998a y García-Huidobro, 1999) y que reúne los esfuerzos oficiales por delinear un programa integral en el campo de la formación inicial y continua. La segunda vertiente está referida a la definición específica de las "Funciones y las competencias necesarias para ejercerlas", y constituyen la base sobre la cual se generan los acuerdos para la definición de un Marco para la Buena Enseñanza (MBE) (Mineduc, 2003) y la entrada en vigencia del sistema de evaluación de los profesores. La tercera dice relación con las actuales iniciativas tendientes a lograr acuerdos sobre la construcción de una "Carrera profesional".

\section{b. Categoría $N^{o}$ 2. El modelo profesional reflexivo}

En las propuestas educativas se encuentran, desde inicios de años 90', alusiones al modelo profesional y reflexivo. Este modelo asume la forma de un referente para los cambios que se proponen al currículo de formación de los profesores de secundaria. Se señala por ejemplo:

"Más allá de las diferencias de enfoques existentes respecto de la profesionalización, lo central de estas demandas se puede sintetizar en la necesidad de pasar de un modelo funcionario (o uno técnico), donde el fuerte de la actividad estaba en la aplicación de normas (o técnicas), a un modelo de ejercicio autónomo, en donde el profesor tiene la capacidad para interpretar situaciones, proponer y desarrollar cursos de acción consecuentes, adecuados al contexto y en colaboración con otros profesionales" (Gysling, 1994: 97).

En el discurso oficial, "la reflexión" constituye el eje central sobre el cual es posible distinguir lo particular del modelo. El Mineduc reconoce, además, que se trata de una capacidad inherente. Es decir, un profesor de secundaria que se conciba:

"Como un sujeto que construye conocimiento no sólo desde la apropiación crítica de lo construido por otros, sino que también a partir de la reflexión individual/colectiva acerca de su acción docente” (Noguera, et al., 2002: 13).

Dando un paso más allá del reconocimiento y de sus características, el MBE del año 2003 establece que, siendo inherente a la condición de profesionalidad, la reflexión constituye un ámbito o dimensión fundamental dentro de las "Responsabilidades profesionales". 


\section{c. Categoría $N^{o}$ 3. Competencias del profesional reflexivo}

El MBE es sin duda el referente oficial para dar cuenta de las competencias del profesor. Al respecto, las autoridades identifican cuatro dimensiones o áreas de competencias: Preparación de la enseñanza, Creación de un ambiente propicio para el aprendizaje, Enseñanza para el aprendizaje de todos los alumnos y Responsabilidades profesionales. En este documento, se advierte la preponderancia que adquieren las competencias didácticas.

Las referencias a este dominio están presentes también en la mayor parte de los documentos oficiales analizados. Cuando se alude a lo que significa ser un "buen profesor" se señala por ejemplo: "utilizar una diversidad de estrategias y una diversidad de medios si se pretende desarrollar un proceso eficaz de aprendizaje" (MINEDUC. MECE-Media, 1997: 18).

Entre las tareas demandadas por el Mineduc a todos los profesores chilenos en el año 2000, es posible identificar el lugar primordial dado a la "implementación de nuevas metodologías y elaboración de nuevos recursos didácticos". Al respecto, según las autoridades, esto implicaría contar con las siguientes competencias:

a. Capacidad para desarrollar y evaluar material didáctico.

b. Manejo de nuevas metodologías.

c. Manejo de procesos de apoyo del aprendizaje.

d. Proponer y desarrollar cursos de acción consecuentes y adecuados al contexto.

e. Realizar ensayos de nuevas acciones en el aula.

\section{d. Categoría $N^{o}$ 4. Los desafios del profesor}

La identificación de los actuales desafíos educativos constituye para las autoridades un elemento de definición. En primer lugar, porque permiten apreciar el giro en las demandas que debe enfrentar el sistema educativo, y en segundo lugar, porque permiten proyectar las competencias que será necesario evaluar y promover entre los profesores (Mineduc, 2008) En esta dirección, los principales desafíos que se plantean hoy al profesor de secundaria son: "desarrollar una posición activa ante la expansión del conocimiento científico-tecnológico", "diseñar estrategias didácticas innovadoras", "usar eficazmente del currículo" y "mejorar sus competencias".

\section{EN SÍNTESIS}

Las actuales políticas educativas referidas a la formación y el desarrollo profesional de los profesores en ejercicio promueven el desarrollo de una identidad "profesional" que define al profesor de secundaria sobre la base de los siguientes elementos:

Un profesor como experto y responsable de la enseñanza y el aprendizaje de los alumnos. Las consideraciones didácticas son superlativas. Su función, por lo tanto, no es la transmisión sino la mediación.

Un profesor capaz de adaptarse a la incertidumbre y de responder innovadoramente a los desafíos que ella implica. El motor fundamental de estos procesos es la reflexión.

La preponderancia de las competencias didácticas permite sostener que los nuevos referentes propuestos exigen de los profesores una recomposición de su identidad en 
torno a una combinación de postulados del modelo "práctico reflexivo" desarrollados originalmente por Schön (1987).

\subsection{IDENTIDAD PROFESIONAL DE LOS PROFESORES DE SECUNDARIA}

\section{a. Modelo de referencia en la identidad profesional de los profesores de secundaria}

El gráfico 1 muestra que la mayor identificación de los profesores es con el modelo práctico $(3,55)$. En segundo lugar, y con mínimas diferencias, es con el modelo crítico $(3,49) \mathrm{y}$, en tercer lugar, con el modelo técnico $(2,86)$.

\section{Gráfico 1}

Modelo de Identificación para los profesores

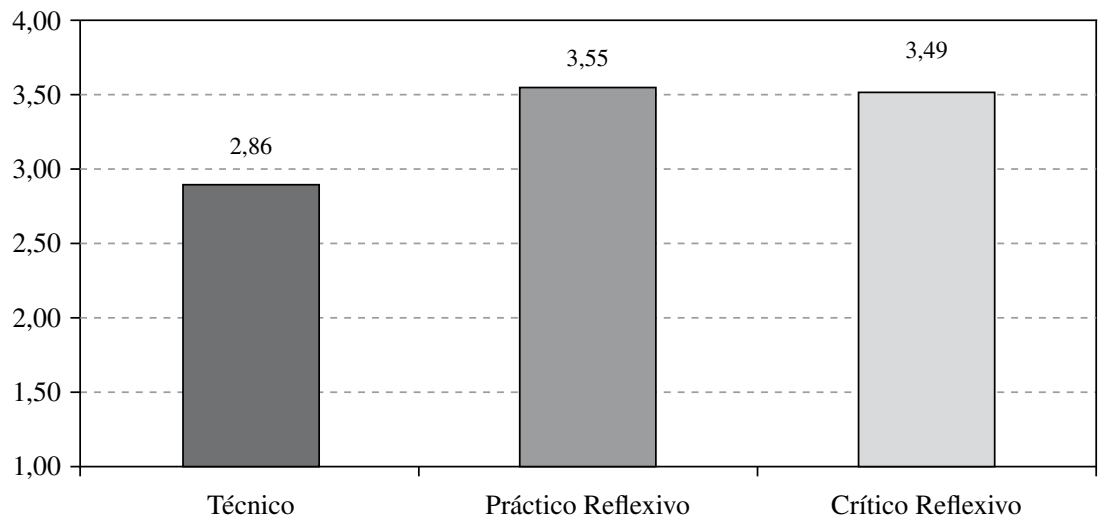

Como se observa en la Figura 3, los resultados obtenidos para el total de profesores $(n=64)$ muestran cómo se comportan los promedios de identificación con los tres modelos identitarios. Las mínimas diferencias entre las medias y las medianas permiten sostener la existencia de una distribución normal en las identificaciones, lo que es corroborado además por la "moderada" desviación en cada caso. En este sentido, cabría señalar que la media es un indicador que no se encuentra subestimado y que las tendencias de identificación no se encuentran distorsionadas.

Para evaluar la sustentabilidad de los promedios obtenidos por profesores participantes que pertenecen a tres liceos de distinto tamaño se ha recurrido a la prueba $t$. El resultado obtenido (67,4 y 83,6 respectivamente), posible de ser observado en el Figura 4, permite señalar que las diferencias de medias $(2,86$ y 3,52) entre un modelo "técnico" y aquél de carácter "profesional y reflexivo" (práctico y crítico) son significativas con un $95 \%$ de confiabilidad.

Por lo tanto, es posible establecer que los profesores participantes definen su identidad profesional fundamentalmente a través de la imagen de un profesional "reflexivo" y "práctico". Sin embargo, es posible plantear además que, si bien la apreciación de los 
Figura 3

Análisis descriptivo de identificación con los modelos

\begin{tabular}{|l|c|c|c|}
\cline { 2 - 4 } \multicolumn{1}{c|}{} & Modelo Técnico & Práctico Reflexivo & Reflexivo Crítico \\
\hline No válida & 64 & 64 & 64 \\
\hline Faltante & 0 & 0 & 0 \\
\hline Media & 2,8600 & 3,5508 & 3,4935 \\
\hline Mediana & 2,8333 & 3,5833 & 3,5833 \\
\hline Moda & 2,50 & 3,33 & 3,58 \\
\hline Desviación estándar &, 33641 &, 34587 &, 37069 \\
\hline Mínima & 2,21 & 1,92 & 1,75 \\
\hline Máxima & 3,67 & 4,00 & 4,00 \\
\hline
\end{tabular}

Figura 4

Prueba de significación para las medias

Prueba para una muestra

\begin{tabular}{|c|c|c|c|c|c|c|}
\hline & \multicolumn{6}{|c|}{ valor de prueba $=0$} \\
\hline & \multirow{2}{*}{$\mathrm{t}$} & \multirow{2}{*}{ gl } & \multirow{2}{*}{$\begin{array}{c}\text { Sig. } \\
\text { (bilateral) }\end{array}$} & \multirow{2}{*}{$\begin{array}{l}\text { Diferencia } \\
\text { de medias }\end{array}$} & \multicolumn{2}{|c|}{$\begin{array}{l}95 \% \text { Intervalo de } \\
\text { confianza para la diferencia }\end{array}$} \\
\hline & & & & & Inferior & Superior \\
\hline IP Técnico & 67,461 & 63 & ,000 & 2,86406 & 2,7792 & 2,9489 \\
\hline IP Reflexivo & 83,681 & 63 & ,000 & 3,52969 & 3,4454 & 3,6140 \\
\hline
\end{tabular}

profesores está asociada a una débil identificación con el modelo "técnico", éste no está ausente. Como es posible observar en el gráfico 2, los profesores se identifican fuertemente con sus dimensiones disciplinar y didáctica.

Ha sido dentro del modelo técnico, y especialmente en su relación con la variable "experiencia" donde se han encontrado las diferencias más "significativas". Por ejemplo, llama la atención la constatación de que el aumento de la experiencia parece acentuar la fuerza de identificación con el modelo "técnico", sobre todo en los profesores con más de 30 años (Gráfico 3).

Según se observa en la Figura 5, la variable experiencia, ha sido entre las estudiadas (sexo, liceo, disciplina, etc.) aquélla que produce las diferencias más significativas.

La identificación con el modelo "técnico" conduce a pensar en la posibilidad de variables de orden contextual-cultural, es decir que al interior de los liceos aún persiste la demanda y realización de tareas (sobre todo de orden "didáctico" o "disciplinar") que tienen, por principios orientadores de su definición, los contenidos de dicho modelo. 


\section{Gráfico 2}

Modelos identitarios. Cruce de dimensiones de competencias
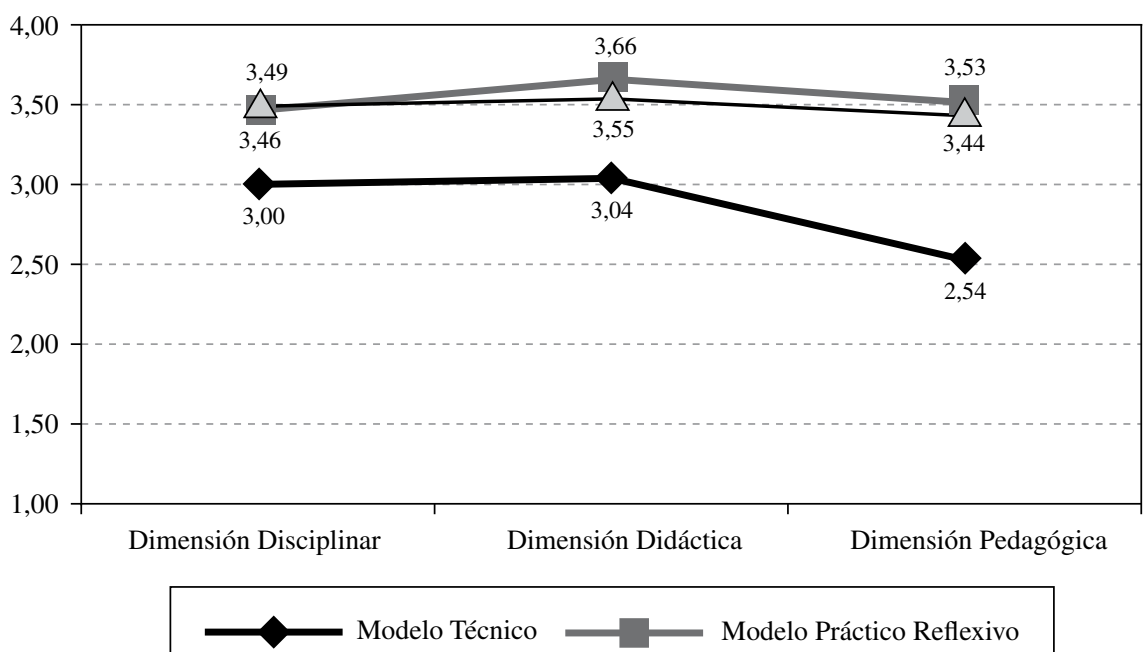

Modelo Crítico Reflexivo

Gráfico 3

Modelos Técnicos. Variación de la identificación según la experiencia

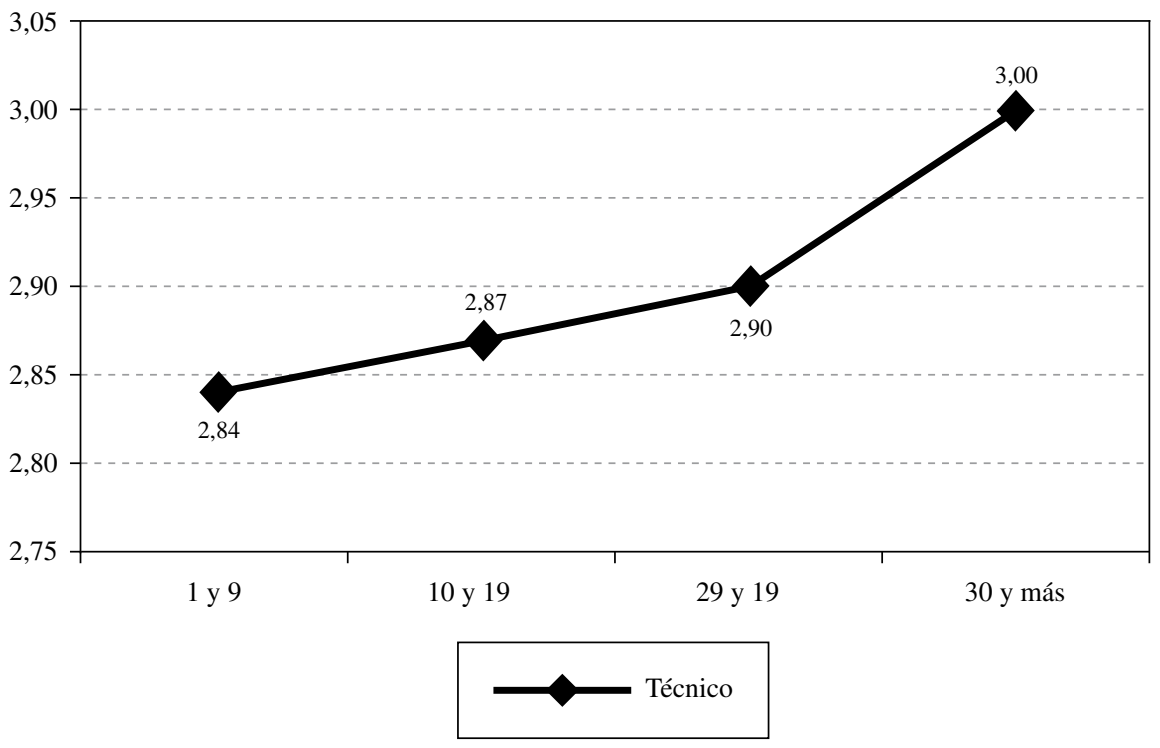




\section{Figura 5}

Incidencia de la variable experiencia sobre la varianza de identificación con el modelo técnico (IT)

\begin{tabular}{|l|c|r|r|r|r|}
\cline { 2 - 6 } \multicolumn{1}{c|}{} & Type III Sun of Squares & df & Mean Square & \multicolumn{1}{c|}{ F } & Sig. \\
\hline Modelo Corregido & $1,457^{\mathrm{a}}$ & 3 &, 486 & 5,138 &, 003 \\
\hline Intercepta & 514,064 & 1 & 514,064 & 5437,403 &, 000 \\
\hline Experiencia & 1,457 & 3 &, 486 & 5,138 &, 003 \\
\hline Error & 5,673 & 60 &, 095 & & \\
\hline Total & 530,634 & 64 & & & \\
\hline Total Corregido & 7,130 & 63 & & & \\
\hline
\end{tabular}

a R Squared = ,204 (Adjusted R Squared = ,165).

b. Ejes y contenidos de la identidad profesional social de los profesores de secundaria

La identidad profesional alude también al sentido de pertenencia y al proceso de verse a sí mismo formando parte de un grupo social poseedor de ciertas características y competencias. En este contexto, las respuestas a la pregunta referida a los contenidos en torno a los cuales se construye la identidad profesional de los profesores de secundaria (sintetizados en la figura 6) se dirigen a identificar, en primer lugar, aquello que se ha definido como "Finalidades del ejercicio" (56,3\%); en segundo lugar, "Las competencias para un buen desempeño" (23,4\%) y en tercer lugar, "Actitudes requeridas" (9,4\%). Las respuestas restantes se han distribuido con menores porcentajes en torno a: "Demandas sociales" y "Problemáticas docentes actuales".

Figura 6

Tres principales elementos que identifican a los profesores de secundaria

\begin{tabular}{|c|c|c|c|c|}
\hline Identifican & Profesores & $\%$ & Contenidos asociados & Profesores \\
\hline \multirow{3}{*}{$\begin{array}{l}\text { En primer lugar: } \\
\text { La finalidad del } \\
\text { ejercicio }\end{array}$} & \multirow{3}{*}{36} & \multirow{3}{*}{56,3} & 1. Formar en valores y la personalidad & 22 \\
\hline & & & 2. Preparación académica & 8 \\
\hline & & & 3. Cultura general & 6 \\
\hline \multirow{3}{*}{$\begin{array}{l}\text { En segundo lugar: } \\
\text { Lo que requiere } \\
\text { saber-hacer }\end{array}$} & \multirow[t]{3}{*}{15} & \multirow[t]{3}{*}{23,4} & 1. Disciplina (contenidos asignatura) & 10 \\
\hline & & & 2. Didácticos (estrategias y recursos) & 3 \\
\hline & & & 3. Pedagógicos (relacionales) & 2 \\
\hline \multirow{3}{*}{$\begin{array}{l}\text { En tercer lugar: } \\
\text { Las actitudes } \\
\text { requeridas }\end{array}$} & \multirow[t]{3}{*}{6} & \multirow[t]{3}{*}{9,4} & 1. Apertura/recepción & 3 \\
\hline & & & 2. Compromiso & 2 \\
\hline & & & 3. Credibilidad & 1 \\
\hline
\end{tabular}


Un aspecto interesante en estos resultados ha sido la constatación de que el eje fundamental de identificación para los profesores de secundaria no es, como podría esperarse luego de una larga formación o como resultado de las recientes reformas, la posesión de un conjunto de competencias disciplinares o didácticas, éstas ocupan un lugar secundario. Por el contrario, el eje de identificación está asociado a las finalidades de formación "valórica o de desarrollo de la personalidad integral de los alumnos" (34,3\%). Este resultado es interesante porque invita a pensar que, de forma independiente del contenido que se enseña y el cómo se enseña, los profesores están abocados a desplegar aquellas competencias socio-afectivas que les permitan atender las demandas cotidianas y personales de sus alumnos. A nuestro juicio, esto releva además "el carácter o naturaleza fundamentalmente social y relacional que asume la construcción de la identidad profesional.

\section{EN SÍNTESIS}

Los profesores se identifican totalmente con los principios de un modelo profesional, aunque específicamente con el modelo práctico reflexivo. Sin embargo, esta identificación es compleja y dinámica, porque no significa que ellos no compartan contenidos de otro modelo. Los profesores con mayor experiencia, por ejemplo, sostienen sin mayores cuestionamientos una coexistencia de referentes identitarios prácticos y técnicos. $\mathrm{Al}$ respecto, esto puede ser el resultado de la consolidación de una estrategia que les permite atender las diversas y a veces contradictorias demandas, lo que se ajusta o articula bastante bien con la hipótesis de existencia de una identidad profesional multidimensional o plural y de un núcleo de identidad.

Esta coexistencia de referentes para la identidad profesional confirma los hallazgos realizados por investigadores europeos a fines de los 90', específicamente aquellos obtenidos por Piot (1997) quien señaló que las representaciones de los profesores de secundaria no parecen ordenarse exclusivamente detrás de un solo modelo, aunque sí es posible establecer referentes dominantes. Para los profesores parece no existir contradicción, porque a diferencia de un modelo teórico que se valida en supuestos epistemológicos, los principios subyacentes a las competencias que movilizan en la práctica se validan en razón de su utilidad para resolver y atender los problemas. Por consecuencia, no hay pérdida de conciencia o de unidad porque para ello, según sostienen Dubet (2002), Sainsaulieu (1996) y Coldron y Smith (1999), entre otros, sería necesaria la vivencia de un profundo conflicto biográfico.

\section{CONCLUSIONES}

Que el modelo del práctico reflexivo se constituya en el marco de referencia o de identificación primordial para la identidad profesional de los profesores sugiere un éxito de las políticas educativas, específicamente a nivel de la instalación de una nueva categoría de conceptualización del rol. Sin embargo, y en un hecho muy interesante, cabe advertir que la identificación de los profesores con este modelo no es exclusiva ni excluyente, lo que invita a reflexionar sobre la articulación existente entre las adopciones epistemológicas bajo las cuales se diseñan las políticas y la lógica o naturaleza de las tareas a las cuales están abocados en forma cotidiana los profesores. 
Si bien existe una coincidencia de identificación con el modelo práctico reflexivo, al mismo tiempo se subestima o desconoce el contenido bajo el cual los profesores construyen su identidad, porque mientras las autoridades educativas se esfuerzan en promover competencias didácticas, los profesores movilizan competencias destinadas a satisfacer los desafíos socio-afectivos que les presentan sus jóvenes estudiantes.

Por lo tanto, puede aludirse a un choque epistemológico entre una política educativa que se fundamenta científicamente y una epistemología de lo cotidiano en los profesores, que a diferencia de aquélla, se fundamenta bajo criterios de tradición y factibilidad. Pero los resultados no solo confirman diferencias en el contenido de identificación con el modelo práctico, sino que además a nivel de la dinámica de su constitución. Mientras la identidad de los profesores se construye a partir de la coexistencia e integración, aquélla promovida por las políticas educativas lo hace a partir de la exclusión y sustitución de otros contenidos.

Cabe preguntarse entonces por la viabilidad y pertinencia de las innovaciones o cambios educativos que se proyectan a partir de una identidad profesional concebida como una construcción que se realiza sobre dimensiones y contenidos únicos y excluyentes. En Chile, un ejemplo está dado por los programas de formación permanente. Estos son diseñados e implementados sin consideración de las necesidades reales y, menos aún, de las definiciones o creencias. En definitiva, los profesores son considerados como un grupo homogéneo.

$\mathrm{Al}$ respecto, que las mayores diferencias encontradas se expliquen por la experiencia permite poner en duda la efectividad de estos programas para promover las competencias necesarias para mejorar la calidad de la enseñanza y de la educación. Si la identidad es una definición a partir del cual el profesor proyecta su desarrollo como profesional, parece más pertinente proyectar el diseño de políticas de formación de profesores en servicio bajo la consideración de las necesidades, contenidos y etapas biográficas por las cuales ellos atraviesan.

Este análisis es coincidente con las recomendaciones del Teaching And Learning International Survey (TALIS, 2009), específicamente sobre la efectividad de las políticas de formación de los profesores en servicio cuando no se consideran variables asociadas a las definiciones, creencias y conocimientos previos del profesor. Se advierte que los gobiernos incurren en importantes gastos financieros, pero que no producen cambio alguno en las prácticas de los profesores porque no logran modificar el núcleo de sus identidades.

Las características de la investigación desarrollada impiden extrapolar sus resultados a contextos más amplios, sin embargo, existe la convicción de que pueden constituir un excelente punto de partida para discutir sobre la importancia de los procesos identitarios en el cambio educativo.

\section{REFERENCIAS BIBLIOGRÁFICAS}

Astolfi, J. (2003). Éducation et formation: nouvelles questions, nouveaux métiers. ESF Editeur: Issy-Les-Moulineaux.

Astolfi, J. (2004). Savoirs en action et acteurs de la formation. Rouen: Publications de l'Université de Rouen. 
Beauchamp, C. (2009). Understanding teacher identity: an overview of issues in the literature and implications for teacher education. Cambridge Journal of Education. Vol. 39, № 2. 175-189.

Beijaard, D. et al. (2000). Teachers' perceptions of professional identity: an exploratory study from personal knowledge. Teaching and Teacher Education. Vol. 16, № 7. pp. 749-764.

Berger, P. y Luckmann, T. (1986). La construction sociale de la réalité. Meridiens Klincksieck, Paris, 1992 (P. Taminiaux, Trad.)

Bolivar, A. (1999). Ciclo de vida profesional del profesorado de secundaria. Desarrollo personal y formación. Bilbao: Ediciones Mensajero.

Cattonar, B. (2001). Les identités professionnelles enseignantes. Ébauche d'un cadre d'analyse. Cahiers de Recherche du Girsef. Vol. 10, pp. 1-34 (UCL, Belgique).

CIDEC (1999). Competencias profesionales: enfoques y modelos a debate. Cuaderno de trabajo $N^{\circ}$ 27. España.

Coldron, J. y Smith, R. (1999). Active location in teachers' construction of their professional identities. Journal of Curriculum Studies. Vol. 19, No 6, pp. 711-726.

Czop, L. (2008). Professional identity of a reading teacher: responding to high-stakes testing pressures. Teachers and teaching: theory and practice. Vol. 14, $\mathrm{N}^{\circ}$ 3, pp. 239-252.

Day, C., Elliot, B. \& Kington, A. (2005). Reform, standards and teacher identity: Challenges of sustaining commitment. Teaching and Teacher Education 21 (2005) 563-577.

Desjardins, J. et al. (2006). Développer des compétences en enseignement. Quelle place pour la réflexión profesionnelle? Montréal, Québec: Éditions de l'ACFAS.

Dubar, C. (2000). La Crise des identités. L'interprétation d'une mutation. Paris. Ed. Le Lien Social).

Galaz, A. (2010). Competencias para una enseñanza efectiva de las ciencias. En: Cofré, H. (Editor). Cómo mejorar la enseñanza de las ciencias en Chile: Perspectivas internacionales y desafíos nacionales. Santiago: Ediciones UCSH.

Hong, J. (2010) Pre-service and beginning teachers' professional identity and its relation to dropping out of the profession. Teaching and Teacher education. 26 1530-1543.

Johnson, R. B. y Turner, L. A. (2003). Data collection strategies in mixed methods research. In A. Tashakkori, \& C. Teddile (Eds.), Handbook of mixed methods in social and behavioral research (pp. 297e319). Thousand Oaks, CA: Sage.

Kelchtermans, G. (2001). Formation des enseignants. L'apprentissage réflexif à partir de la biographie et du contexte. Recherche et Formation Vol. 36, pp. 43-67.

Lasky, S. (2005). A Sociocultural approach to understanding teacher identity, agency and professional vulnerability in a context of secondary school reform. Teaching and Teacher Education 21 (8), 899-916.

Letor, C. (2003). l'accès aux compétences est-il plus (ini)équitable que l'accès aux saviors traditionnels? Cahier de Recherche du Girsef Vol. 25, pp. 1-20.

Maroy, C. (2001). Le modèle du praticien réflexif à l'épreuve de l'enquête. Les Cahiers de Recherche $d u$ Girsef, Vol. 12, pp. 2-26 UCL: Belgique.

Maroy, C. (2005). Les évolutions de travail enseignant en Europe. Facteurs de changement, incidences et résistances. Les Cahiers de Recherche en Education et Formation du Girsef, Vol. 42. pp. 2-35. UCL: Belgique.

MINEDUC (1998). La Reforma en marcha. (Santiago, MINEDUC)).

MINEDUC (1999). Programa de Fortalecimiento de la Formación Inicial Docente (PFFID): Profesores para el Siglo XXI. Santiago: MINEDUC.

MINEDUC (2003). Marco para la Buena Enseñanza. Santiago: MINEDUC.

MINEDUC (2004). Compendio de información estadística. Santiago: MINEDUC.

MINEDUC. MECE-MEDIA (1996). Mejorando el aprendizaje de nuestros alumnos. Manual para los Grupos Profesionales de Trabajo. Chile. 
Noguera, M.I. et al. (2002). Desarrollo Profesional Docente. Experiencias de colaboración en Enseñanza Media. Santiago: MINEDUC.

Paquay, L. et al. (Dir.) (2001). Former des enseignants professionnels. Quelles stratégies? Quelles compétences? Bruxelles: De Boeck.

Sainsaulieu, R. (1996). L'identité et les relations de travail. En: Éducation Permanente, Vol. 3, $\mathrm{N}^{\circ} 128$, pp. $187-206$.

Schön, D. (1983). The reflective practitioner: how professionals think in action. New York: Basic Books.

Schön, D. (1987). Educating the reflective practitioner. Toward a new design for teaching and learning in the professions. S. Francisco: Jossey Bass.

Tardif, M. ET Lessard, C. (1999). Le travail enseignant au quotidien. Contribution à l'étude du travail dans les métiers et les professions d'interactions humaines. Les Presses de l'Université de Laval.

Tardif, M. (2004). Los saberes del docente y su desarrollo profesional. Madrid: Narcea.

Tardif, M. (2008). "Développer un programme par compétences: de l'intention à la mise en oeuvre", Pédagogie collégiale, 16(3), pp. 36-45.

Vonck, J.H. (1995). Teacher induction: an essential elemental at the start of teachers. Revista Española de Pedagogía $\mathrm{N}^{\circ}$ 200, pp. 67-91.

Woods, P. Y Jeffrey, B. (2002). The reconstruction of primary teachers' identities. British Journal of Sociology of Education. Vol. 23, $\mathrm{N}^{\circ}$ 1, figura 89-106.

Zeichner, K. (1993). El maestro como profesional reflexivo. Cuadernos de Pedagogía $\mathrm{N}^{\circ} 220$, pp. 44-29.

Zeichner, K. y Liston, D. (1999). Enseñar a reflexionar a los futuros docentes. En: Pérez Gómez et al., Desarrollo profesional Docente: política, investigación y práctica, pp. 505-532. Madrid: Akal Ediciones. 
